\title{
SAND CASTING PROCESS IMPROVEMENT USING LEAN PRINCIPLES
}

\author{
Hari Priya $\mathbf{G}^{\mathbf{1}}$, H.Ramakrishna ${ }^{2}$, Y. Sundara Rajalu ${ }^{3}$ \\ ${ }^{I}$ PG Student, Mtech- MEM, Dept. of IEM, Dayananda Sagar College of Engineering, Bangalore-78 \\ ${ }^{2}$ HOD, Dept. of IEM, Dayananda Sagar College of Engineering, Bangalore-78 \\ ${ }^{3}$ General Manager, Quality \& Operational Excellence, Bharat Fritz Werner Ltd, Bangalore -22
}

\begin{abstract}
Lean manufacturing is often seen as a set of tools that reduce the total cost and improve the quality of manufactured products. The lean management philosophy is one which targets waste reduction in every facet of the manufacturing business; however, only recently have studies linked lean management philosophies with improving environmental sustainability. These studies suggest that lean manufacturing is more than a set of lean tools that can optimize manufacturing efficiencies; it is a process and mindset that needs to be integrated into daily manufacturing systems to achieve sustainability. The foundry industry, as well as manufacturing in general, has significant challenges in the current regulatory and political climate with developing an economically and environmentally sustainable business model. Lean manufacturing has proven itself as a model for both economic sustainability and environmental stewardship. Several recent studies have shown that both lean and green techniques and "zero-waste" policies also lead to reductions in overall cost.
\end{abstract}

This paper reviews the literature and some of the lean principles such as the Value Stream Mapping and Waste elimination by implementing $5 S$ in the organization. This paper also aims at reducing lead time by eliminating stress relieving cycle with extended cooling of castings in the mould and stabilize the sand casting process by rationalization of the material grades.

Keywords: Lean Manufacturing, Zero-waste, Value Stream Mapping, Stress Relieving, Rationalization - ***

\section{INTRODUCTION}

\subsection{No Bake Sand Casting Process}

No Bake is a casting process that uses chemical binders to bond the molding sand. The sand is then transported to the mold fill station in preparation for filling of the mold. A mixer is then used to blend sand with the chemical binder and the catalyst. When the sand exits the mixer, the binder begins the hardening process.

After the compaction process, a rollover process is used to remove the mold from the pattern box. The mould is then readied for handling the molten metal. After a shakeout process, the molded sand is taken away from the casting. Then various procedures follow including the finishing and the sand can be reclaimed by thermal means.

In the No-Bake resin sand casting process, sand molds are created using a wood, metal, or plastic pattern. Sand is mixed with a plastic binder in a high-speed mixer. This sand is deposited into box containing the pattern and all essential gating, risers and chills for pouring.

The sand mixture sets up hard in a few minutes and the mold is removed from the pattern. Cores for forming internal passages in the castings are made using the same process. Cores are carefully placed into the molds. The molds are then closed and are ready for pouring.
The chemical nature of the binders makes this a highly specialized process that has to been handled with considerable expertise and knowledge.[1]

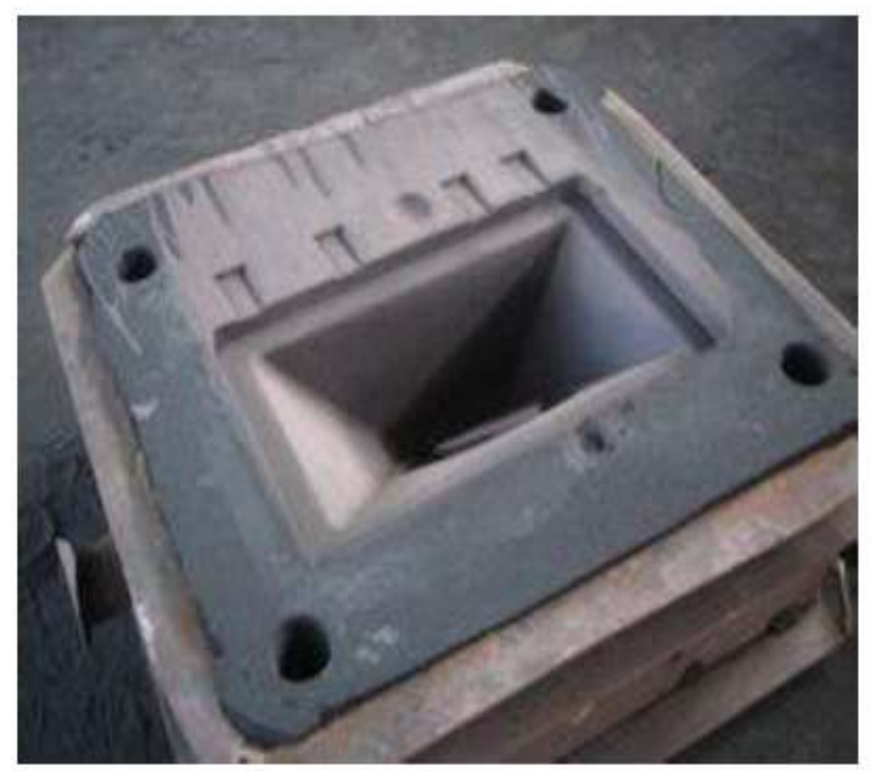

\subsection{Value Stream Mapping}

Value stream mapping is a lean-management method for analyzing the current state and designing a future state for the series of events that take a product or service from its 
beginning through to the customer. At Toyota, it is known as "material and information flow mapping"[2].

\subsection{Stress Relieving}

Stress relieving is carried out on metal products in order to minimise residual stresses in the structure thereby reducing the risk of dimensional changes during further manufacturing or final use of the component.

Machining, and cutting, as well as plastic deformation, will cause a build up of stresses in a material. These stresses could cause unwanted dimension changes if released uncontrolled, for example during a subsequent heat treatment. To minimise stresses after machining and the risk for dimension changes the component can be stress relieved.

Stress relieving is normally done after rough machining, but before final finishing such as polishing or grinding.

Parts that have tight dimensional tolerances, and are going to be further processed, for example by nitrocarburising, must be stress relieved.

Welded structures can be made tension free by stress relieving.

\subsection{Rationalization}

Rationalization means the increase of work process effectiveness on the basis of introducing (rationalizing) arrangements in partial steps of the given activity.

At the rationalizing process it is the improvement of the human activity, increase of its effectiveness; increase of economic efficiency; summary of arrangements for the most effective utilization of work force and technology on the basis of the most modern knowledge of science and technology.
Thus, rationalization means the selection of the right way to the simplification of the production process and process analysis with the goal to reach its purposeful arrangement [3].

\subsection{Methodology}

The methodology followed is the DMAIC

\section{Define Phase:}

\section{Problem Definition}

Structural castings are one of the basic raw material for any of the machine tool industry. The cost and quality of these castings play an important role in the profitability of the business. These castings are produced in their own foundry through No Bake Sand Moulding process. There are six grades of castings , 3 Grey cast iron ( G2, G3, G4) and 3 Spherodical Graphite Iron ( SG1, SG2, SG3) produced using no bake process. Due to the variety of grades there is less stabilization in the production of these castings and more rejections have occurred.

\section{Objectives}

\section{Primary Objective:}

To improve sand casting process in foundry, supply improved and reduced cost castings to the Machine tool Industry.

\section{Secondary Objective:}

- To reduce lead time by Value Stream Mapping

- To rationalize casting grades and improve productivity

- To eliminate Stress Relieving cycle or do stress relieving only once for critical castings

- To reduce design related defects

- To eliminate 7 wastes applying lean principles

\section{Gantt Chart}

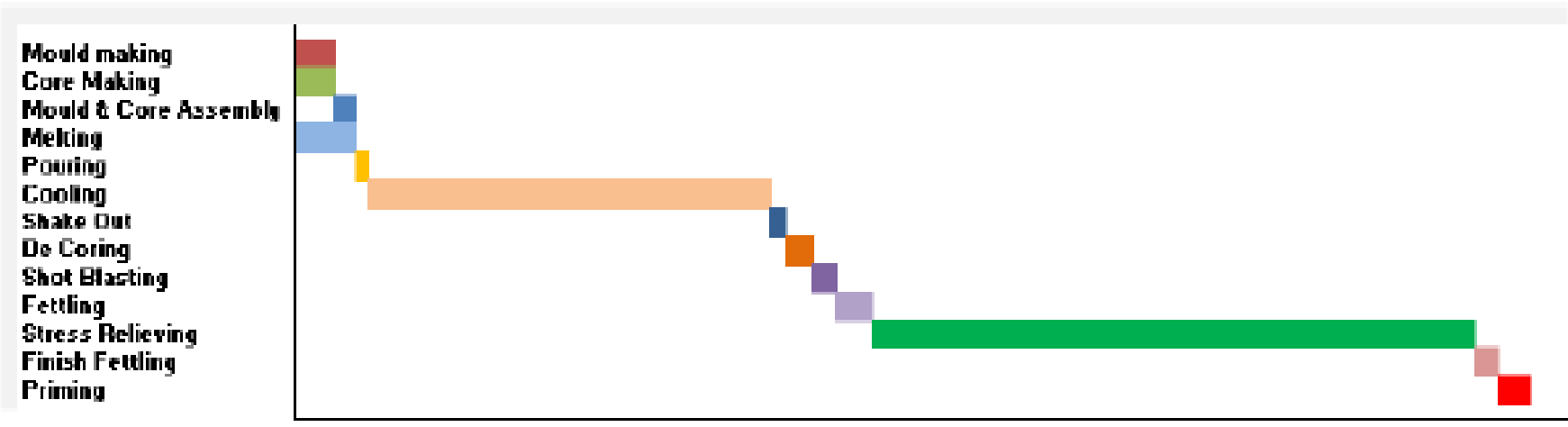




\section{As is Process Map}

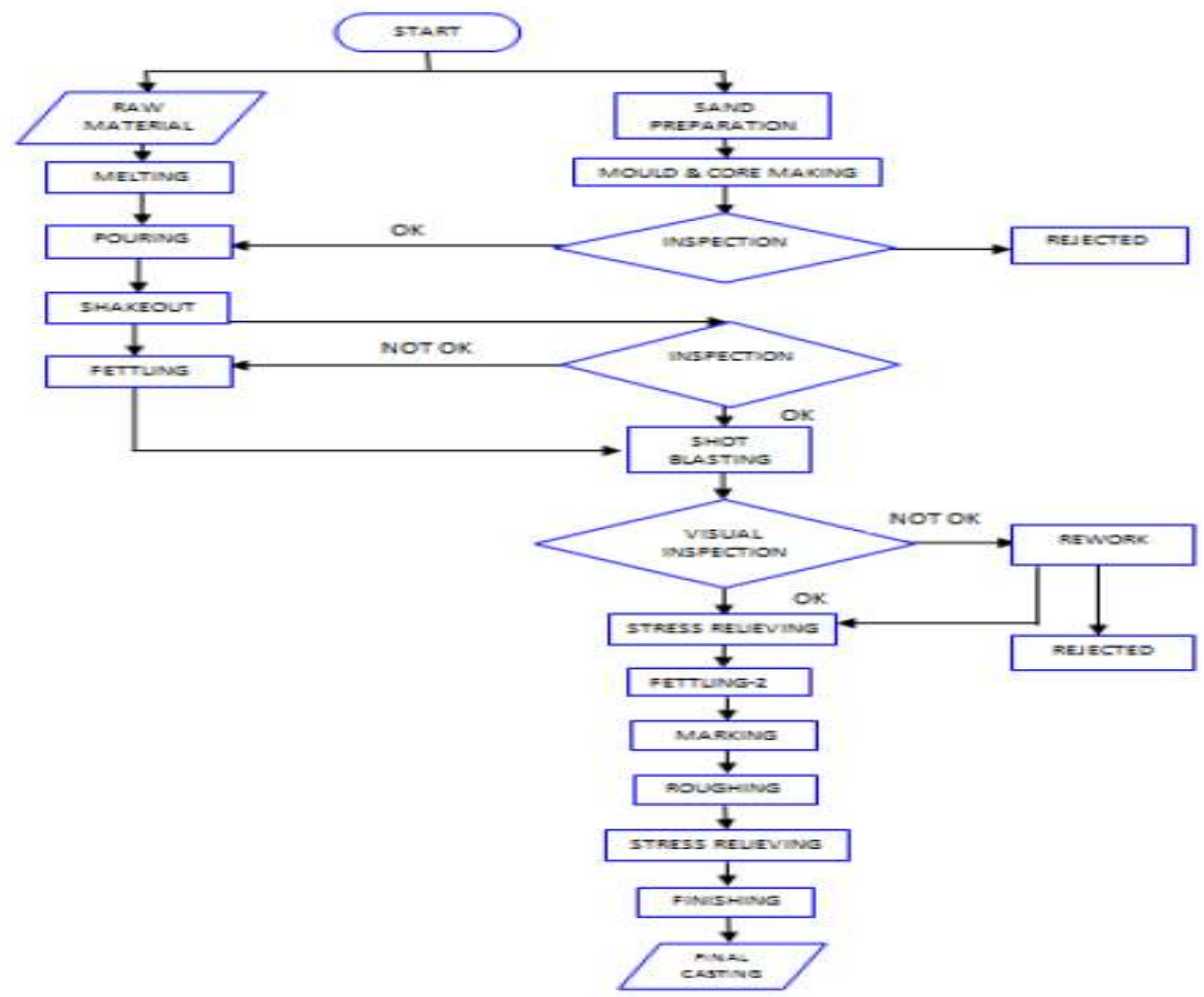

\section{Measure phase}

\section{Current State Value Stream Mapping}

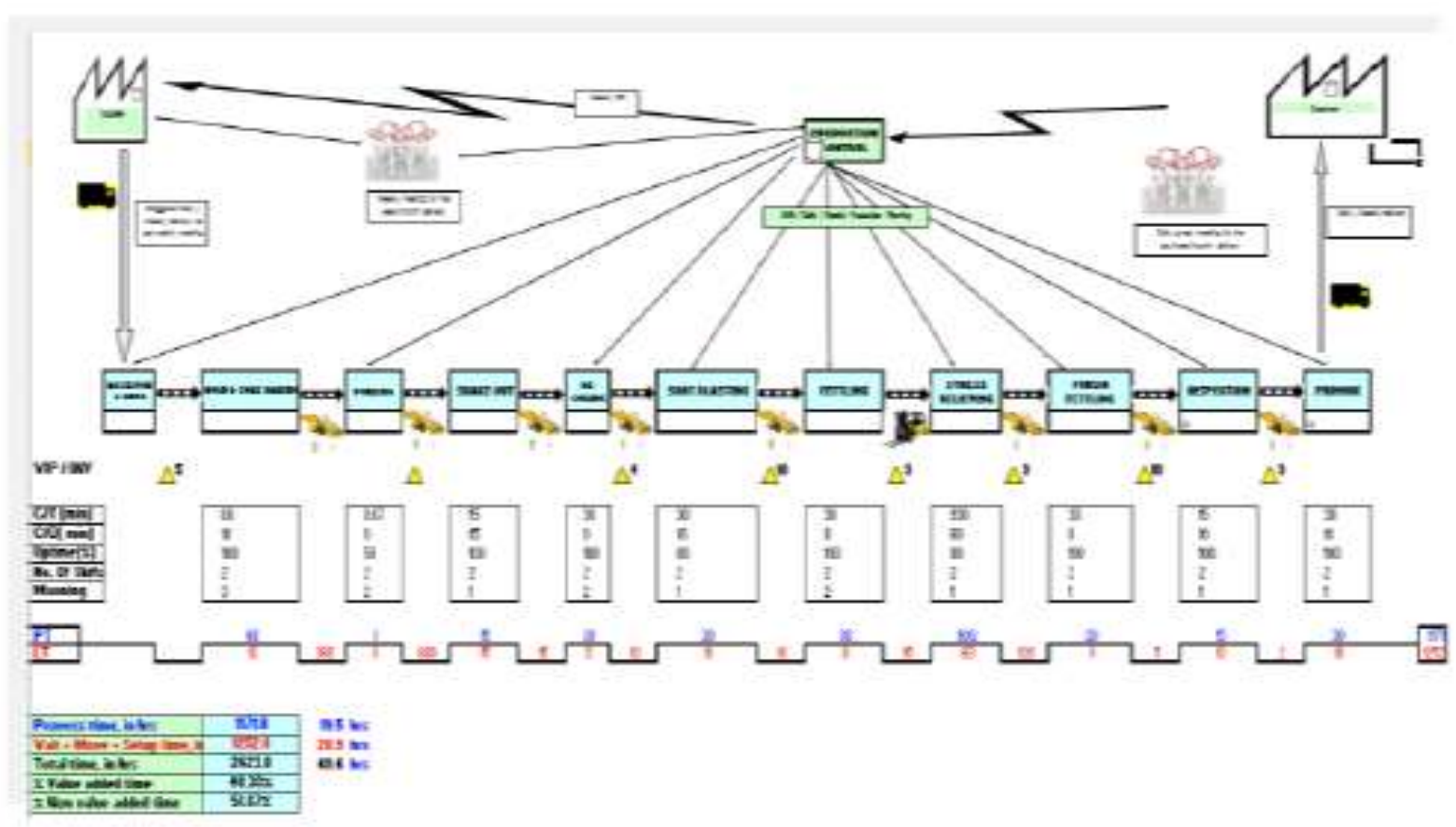




\begin{tabular}{|c|c|}
\hline Process time, in min & 1171.0 \\
\hline Wait + Move + Setup time, in min & 1252.0 \\
\hline Total time, in min & 2423.0 \\
\hline$\%$ Value added time & $48.33 \%$ \\
\hline \% Non value added time & $51.67 \%$ \\
\hline
\end{tabular}

Takt Time calculation

Working shift per day $=2$

Working hours per shift $=91 / 2$ hours

Available time per shift $=570$ minutes

Tea break per shift $=2$ breaks $* 10$ minutes $=20$ minutes

Lunch break per shift $=30$ minutes

Down time per shift $=0$

Net working time per shift $=$ [available time- $($ breaks + break down $)]=[570-(20+30+0)]=520$

Customer demand per day $=20$ Nos or $10000 \mathrm{~kg}$

Takt time $=$ Available production time/Total daily quantity required $=1040 / 20=52$ minutes

\section{Rejection Details}

- Overall casting rejections( For Financial Year Apr 2014- Mar 2015)

\begin{tabular}{|l|r|r|r|}
\hline $\begin{array}{l}\text { Total Supplied } \\
\text { Qty }\end{array}$ & $\mathbf{6 3 6 9}$ & & \\
\hline Sal. \& Rej. Qty & $\mathbf{1 2 5 4}$ & & \\
\hline Percentage & $\mathbf{1 9 . 6 9 \%}$ & 196900 & \\
\hline & & & \\
\hline Salvaged Qty & $\mathbf{9 6 3}$ & & \\
\hline $\begin{array}{l}\text { Salvaged } \\
\text { Percent }\end{array}$ & $\mathbf{1 5 . 1 2 \%}$ & $1,51,200$ & \\
\hline $\begin{array}{l}\text { Rejected Qty } \\
\text { Rejected } \\
\text { Percent }\end{array}$ & $\mathbf{2 9 1}$ & & 2.5 \\
\hline
\end{tabular}

- $\quad$ Type Of Defects Found Gradewise

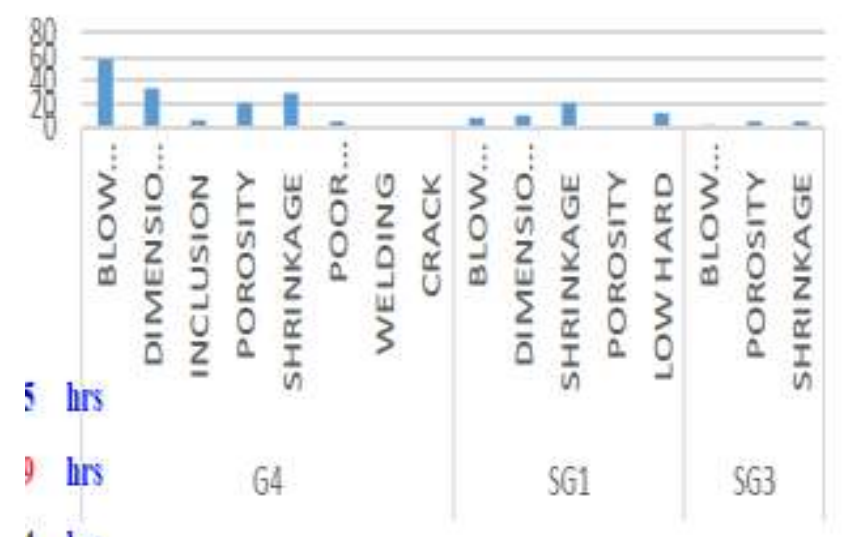

More number of defects are found in grades G4 and SG1 $\checkmark \quad$ Based on the percentage, SG1 stands 1st with more percent of rejections. Since the process of producing castings of grade SG is complicated than producing castings with grade FG.

- Top Components Rejected

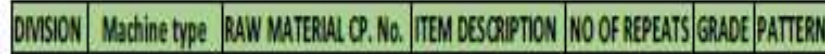

\begin{tabular}{|c|c|c|c|c|c|c|}
\hline WMC & BMUS5 & 00530074503 & Cross slide & 45 & G4 & Woosen \\
\hline WMC & BMW0 & 80530073603 & Table & 16 & G4 & Woosden \\
\hline WMC & TCBSOO & R05506993702 & Table & 12 & G4 & Woosden \\
\hline HMC & TMCicon & P0S50150250 & BEARNG BLCOCX & 12 & 561 & Woosden \\
\hline WMC & BMEO & 105530081904 & Miling head & 11 & G4 & Woosen \\
\hline WMC & & R05596598201 & Cross slide & 10 & G4 & Wooden \\
\hline WMC & BMNS5t & R0O530073400 & Milling Head & 9 & G4 & Wooden \\
\hline HMC & AOOE & POS595925900 & Pallet & 9 & S61. & \\
\hline WMC & Chandat & ro5000012700 & colum & 8 & G4 & Wooden \\
\hline HMC & HIST 3000/4070 & P05596366500 & TABLE & 8 & 561 & Wooden \\
\hline HMC & HSTC & R05596356570 & Table & 8 & 601 & Them: \\
\hline HMC & HSTCW/4070 & R05596506000 & Table & 8 & 661 & \\
\hline VMC & BNBOD & R05330097503 & Table & 6 & 64 & Wooden \\
\hline
\end{tabular}

$\checkmark$ After analysing the rejection data, we found that out of the 5 structural components (Table, Cross slide, Milling Head, Column \& Base) the most rejected components are Table \& Cross slide. Hence these two structural components are considered for study \& experimentation.

\subsection{Analyse Phase}

- TO REDUCE DESIGN RELATED DEFECTS IN THE CASTINGS IN TABLE \& CROSS SLIDE

\begin{tabular}{|c|c|r|}
\hline \multirow{3}{*}{ TABLE } & REJ \% & 8.455 \\
\cline { 2 - 3 } & SAL\% & 4.065 \\
\cline { 2 - 3 } & OVERALL & 12.52 \\
\hline \multirow{2}{*}{$\begin{array}{c}\text { CROSS } \\
\text { SLIDE }\end{array}$} & REJ \% & 1.85 \\
\cline { 2 - 3 } & SAL\% & 9.95 \\
\cline { 2 - 3 } & OVERALL & 11.8 \\
\hline
\end{tabular}

\section{Table}

- The castings of table differ in sizes based on the model of machine tool and its use.

- From the analysis it was found that more rejections are in HSTC table which is a SG casting.

- Other Tables are made of grey iron and the rejections are less compared to HSTC table 


\section{Cross Slide}

- Most of the cross slides were rejected because of the shrinkage in the lead screw bore area which was machined unnecessarily.

\begin{tabular}{|c|c|c|c|}
\hline & $\begin{array}{l}\text { Total } \\
\text { Quantity } \\
\text { Produced }\end{array}$ & $\begin{array}{l}\text { Rejected } \\
\text { Quantity }\end{array}$ & $\begin{array}{l}\text { Percent } \\
\text { Rejection }\end{array}$ \\
\hline HSTC TABLE & 76 & 26 & $34.21 \%$ \\
\hline OTHER TABLE & 776 & 63 & $8.12 \%$ \\
\hline
\end{tabular}

Hence the suggestion for rationalising the grades was arrived and the part nos which are to be rationalised was identified based on their application, size and model.

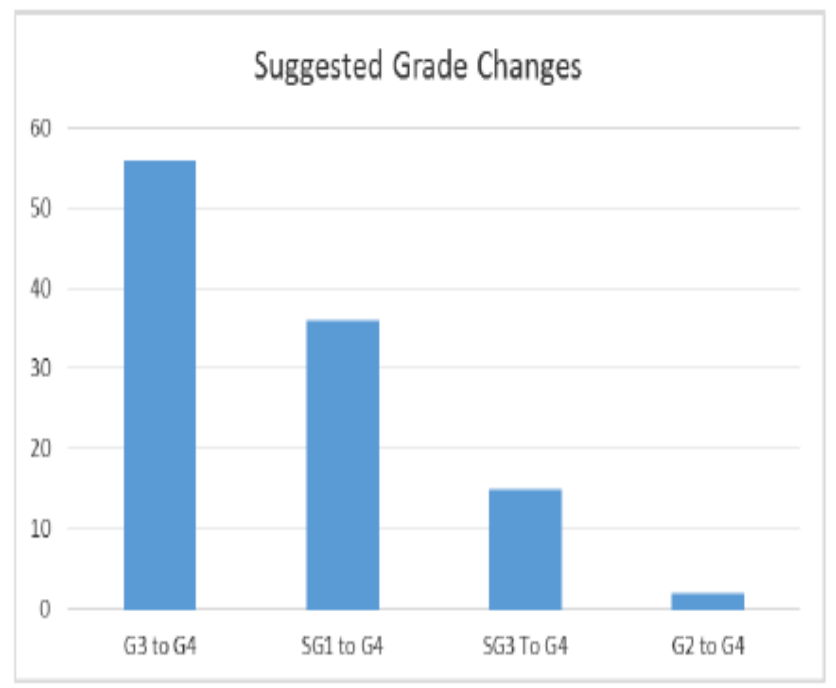

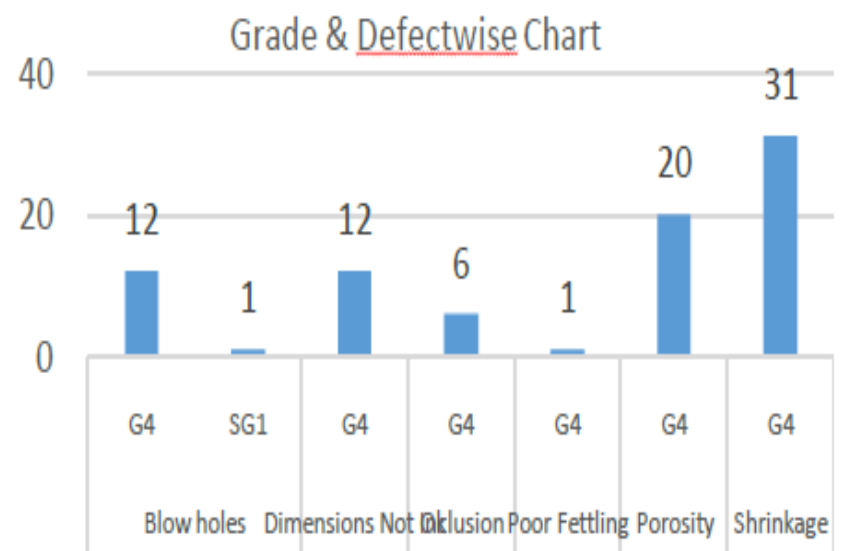

- $\quad$ To reduce this defect shrinkage, chills were introduced and a core with large bore size was made and the machining was eliminated.

\begin{tabular}{|c|c|c|c|c|c|}
\hline MATERIAL NO & Part Description & Machine Type & Total & Material & Design \\
\hline R0530079400 & BMV 45+ MILLING HEAD & BMV 45+ & 333 & $\mathrm{G} 4$ & No $\mathrm{m} / \mathrm{cing}$ at Lead screw area \\
\hline R0506993702 & BMV45+ TABLE & BMV $45+$ & 250 & G4 & No $\mathrm{m} / \mathrm{cing}$ at Lead screw area \\
\hline R0530074503 & BMV45+ CROSS SLIDE & BMV 45+ & 240 & G4 & No $\mathrm{m} / \mathrm{cing}$ at Lead screw area \\
\hline R0530073603 & BMV 60 TABLE & BMV 60 & 173 & G4 & No $\mathrm{m} / \mathrm{cing}$ at Lead screw area \\
\hline R0530081904 & BMV 60 MILLING HEAD & BMV 60 & 154 & G4 & No $\mathrm{m} / \mathrm{cing}$ at Lead screw area \\
\hline R0530149401 & BMV 6O-CROSS SLIDE & BMV 60 & 151 & G4 & No $\mathrm{m} / \mathrm{cing}$ at Lead screw area \\
\hline R0506994002 & BMV45++ CROSS SLIDE & BMV 45++ & 108 & G4 & No $\mathrm{m} / \mathrm{cing}$ at Lead screw area \\
\hline R0537716301 & BMV 51 TABLE & BMV 51 & 96 & G4 & No $\mathrm{m} / \mathrm{cing}$ at Lead screw area \\
\hline R0537743701 & BMV 35-CROSS SLIDE & BMV 35 & 96 & G4 & No $\mathrm{m} /$ cing at Lead screw area \\
\hline R0537741102 & Table BMV 35 & BMV 35 & 94 & G4 & No $\mathrm{m} / \mathrm{cing}$ at Lead screw area \\
\hline R0537744501 & BMV-35MILLING HEAD & BMV 35 & 88 & G4 & No $\mathrm{m} / \mathrm{cing}$ at Lead screw area \\
\hline R0537720503 & BMV51 CROSS SLIDE & BMV 51 & 83 & G4 & No $\mathrm{m} / \mathrm{cing}$ at Lead screw area \\
\hline R0537729603 & BMV 51MILLING HEAD & BMV 51 & 72 & G4 & No $\mathrm{m} / \mathrm{cing}$ at Lead screw area \\
\hline R0530130401 & (VF3OCNC CHANDRA) MILLING HEAD & VF30 CNC CHANDRA & 39 & G4 & No $\mathrm{m} / \mathrm{cing}$ at Lead screw area \\
\hline R0596380700 & CROSSSLIDE HSTC 3070 & HSTC 3070 & 37 & G4 & No $\mathrm{m} / \mathrm{cing}$ at Lead screw area \\
\hline R0596506000 & MILLING HEAD BT 40 HSTC VE & BT 40 HSTC VE & 29 & G4 & No $\mathrm{m} / \mathrm{cing}$ at Lead screw area \\
\hline R0537760602 & BMV 65 MILLING HEAD & BMV 60 & 25 & G4 & No $\mathrm{m} / \mathrm{cing}$ at Lead screw area \\
\hline R0530079601 & BMV $60+$ MILLING HEAD & BMV 60+ & 23 & G4 & No $\mathrm{m} / \mathrm{cing}$ at Lead screw area \\
\hline R0596380800 & CROSS SLIDE HSTC 3050 -VE $\varnothing 32$ BS & HSTC $3050-V E$ & 20 & G4 & No $\mathrm{m} / \mathrm{cing}$ at Lead screw area \\
\hline R0537760203 & BMV 65 TABLE ( $1500 \mathrm{~mm} \times 650 \mathrm{~mm})$ & BMV 65 & 19 & G4 & No $\mathrm{m} / \mathrm{cing}$ at Lead screw area \\
\hline R0530093402 & MILLING HEAD (VF3OCNC VS) & VF 30 CNC VS & 18 & G4 & No $\mathrm{m} / \mathrm{cing}$ at Lead screw area \\
\hline R0530036301 & BMV70 \& 80-MILLING HEAD & BMV $70 \& 80$ & 17 & SG1 & No $\mathrm{m} /$ cing at Lead screw area \\
\hline R0530150901 & BMV45+ RECEIVER (INTEGRAL) & BMV 45+ & 16 & G4 & No $\mathrm{m} / \mathrm{cing}$ at Lead screw area \\
\hline R0530088801 & BMV $60++$ TABLE & BMV $60++$ & 14 & G4 & No $\mathrm{m} / \mathrm{cing}$ at Lead screw area \\
\hline R0530089902 & BMV $60++$ CROSS SLIDE & BMV $60++$ & 14 & G4 & No $\mathrm{m} / \mathrm{cing}$ at Lead screw area \\
\hline R0537760301 & BMV 65 CROSS SLIDE & BMV 65 & 13 & G4 & No $\mathrm{m} / \mathrm{cing}$ at Lead screw area \\
\hline R0596413501 & CROSS SLIDE HSTC- 3050 & HSTC 3050 & 13 & G4 & No $\mathrm{m} / \mathrm{cing}$ at Lead screw area \\
\hline R0596380600 & HSTC $3070 / 4070$ CROSS SLIDE & HSTC $3070 / 4070$ & 12 & G4 & No $\mathrm{m} / \mathrm{cing}$ at Lead screw area \\
\hline
\end{tabular}




\section{To Eliminate Stress Relieving}

\section{Part numbers that undergo stress relieving are identified}

\begin{tabular}{|c|c|c|c|c|c|c|}
\hline Raw Material CP No & Part Description & Machine type & Total & Tonnage & Total Tonnage & Stress Relieved \\
\hline R0530079400 & BMV $45+$ MILLING HEAD & BMV 45+ & 333 & 215 & 71595 & Yes \\
\hline $\mathrm{R} 0506993702$ & BMV45+ TABLE & BMV 45+ & 250 & & & No \\
\hline R0530074503 & BMV45+ CROSS SLIDE & BMV 45+ & 240 & & & No \\
\hline R0530073603 & BMV 60 TABLE & BMV 60 & 173 & & & No \\
\hline R0537740506 & BMV45+ MACHINE BASE & BMV 45+ & 161 & 1035 & 166635 & Yes \\
\hline R0530081904 & BMV 60 MILLING HEAD & BMV 60 & 154 & 425 & 65450 & Yes \\
\hline R0530149401 & BMV 60-CROSS SLIDE & BMV 60 & 151 & & & No \\
\hline R0537740505 & BMV45+ MACHINE BASE & BMV 45+ & 112 & 1035 & 115920 & Yes \\
\hline R0500003901 & KNEE SIZE - 2 & & 111 & 245 & 27195 & Yes \\
\hline R0500079901 & FEED GEAR BOX SIZE - 2 & & 110 & & & No \\
\hline R0506994002 & BMV45++ CROSS SLIDE & BMV 45++ & 108 & & & No \\
\hline R0537716301 & BMV 51 TABLE & BMV 51 & 96 & & & No \\
\hline R0537743701 & BMV 35-CROSS SLIDE & BMV 35 & 96 & 240 & 23040 & Yes \\
\hline R0537741102 & Table BMV 35 & BMV 35 & 94 & & & No \\
\hline R0537744501 & BMV-35MILLING HEAD & BMV 35 & 88 & 135 & 11880 & Yes \\
\hline R0537720503 & BMV51 CROSS SLIDE & BMV 51 & 83 & & & No \\
\hline R0500069000 & TABLE SIZE - 2 & & 80 & 300 & 24000 & Yes \\
\hline R0537729603 & BMV 51MILLING HEAD & BMV 51 & 72 & 395 & 28440 & Yes \\
\hline R0500063300 & H.V. SADDLE SIZE - 2 & & 71 & 148 & 10508 & Yes \\
\hline R0506904205 & TABLE (SURYA / CHANDRA) & SURYA/CHANDRA & 70 & & & No \\
\hline R0500002102 & HORIZONTAL COLUMN SIZE - 2 & & 69 & & & No \\
\hline R0537768600 & BEARING BLOCK BMV 65 & BMV 65 & 57 & 10 & 570 & Yes \\
\hline R0596365800 & HSTC TABLE- 3070 & HSTC 3070 & 54 & 200 & 10800 & Yes \\
\hline R0500029401 & OVER ARM SIZE - 2 & & 52 & & & No \\
\hline R0500064100 & U SADDLE SIZE - 2 & & 50 & 90 & 4500 & Yes \\
\hline R0537740507 & BMV45+ MACHINE BASE & BMV 45+ & 50 & 1035 & 51750 & Yes \\
\hline R0596362400 & HEIGHT BLOCK - 150MM & & 49 & 125 & 6125 & Yes \\
\hline
\end{tabular}

2. Residual stress(Mpa) after and before Stress Relieving

- Residual Stress level in mpa before Stress Relieving

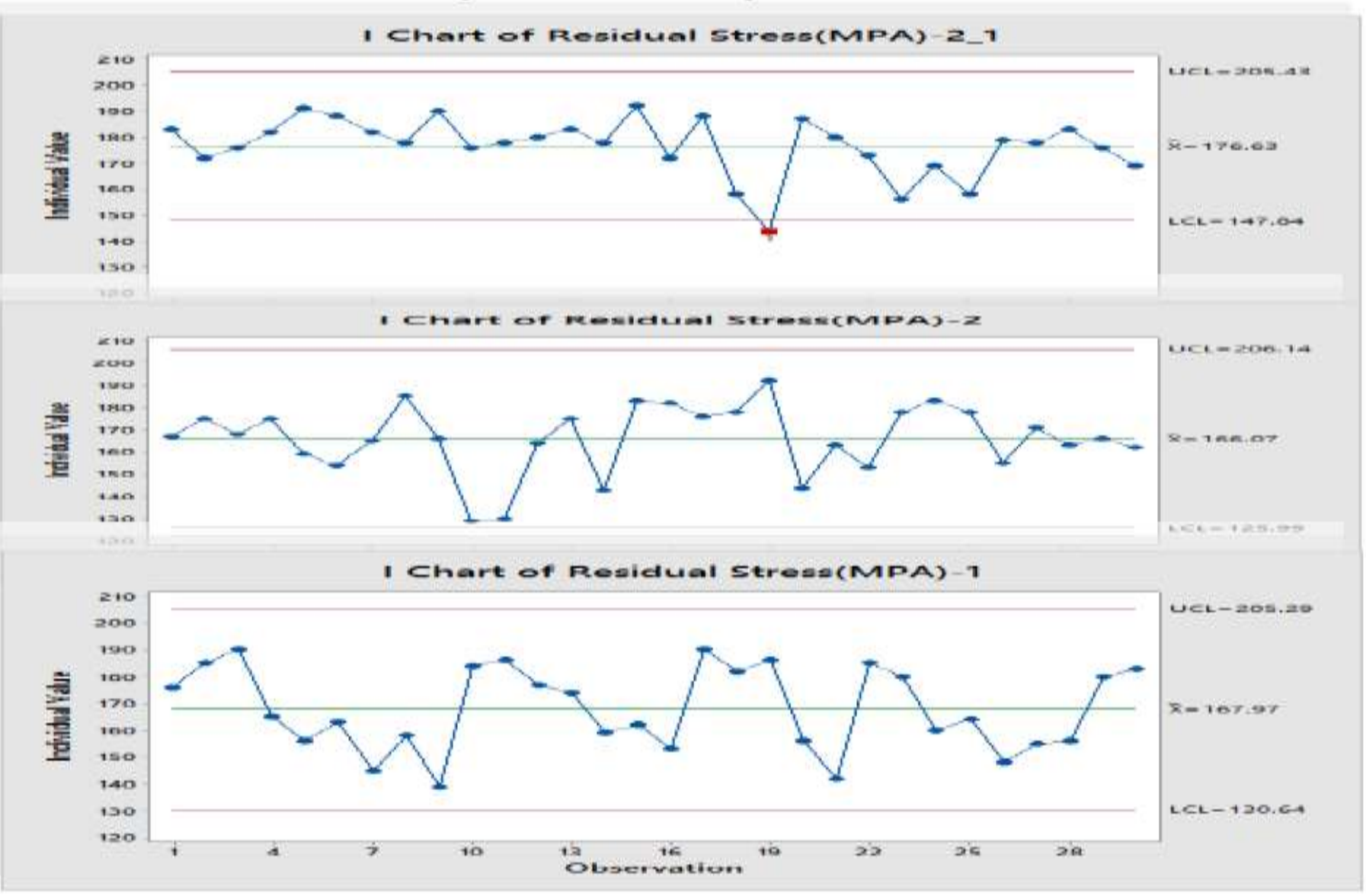


- $\quad$ Residual Stress Level in mpa after Stress Relieving

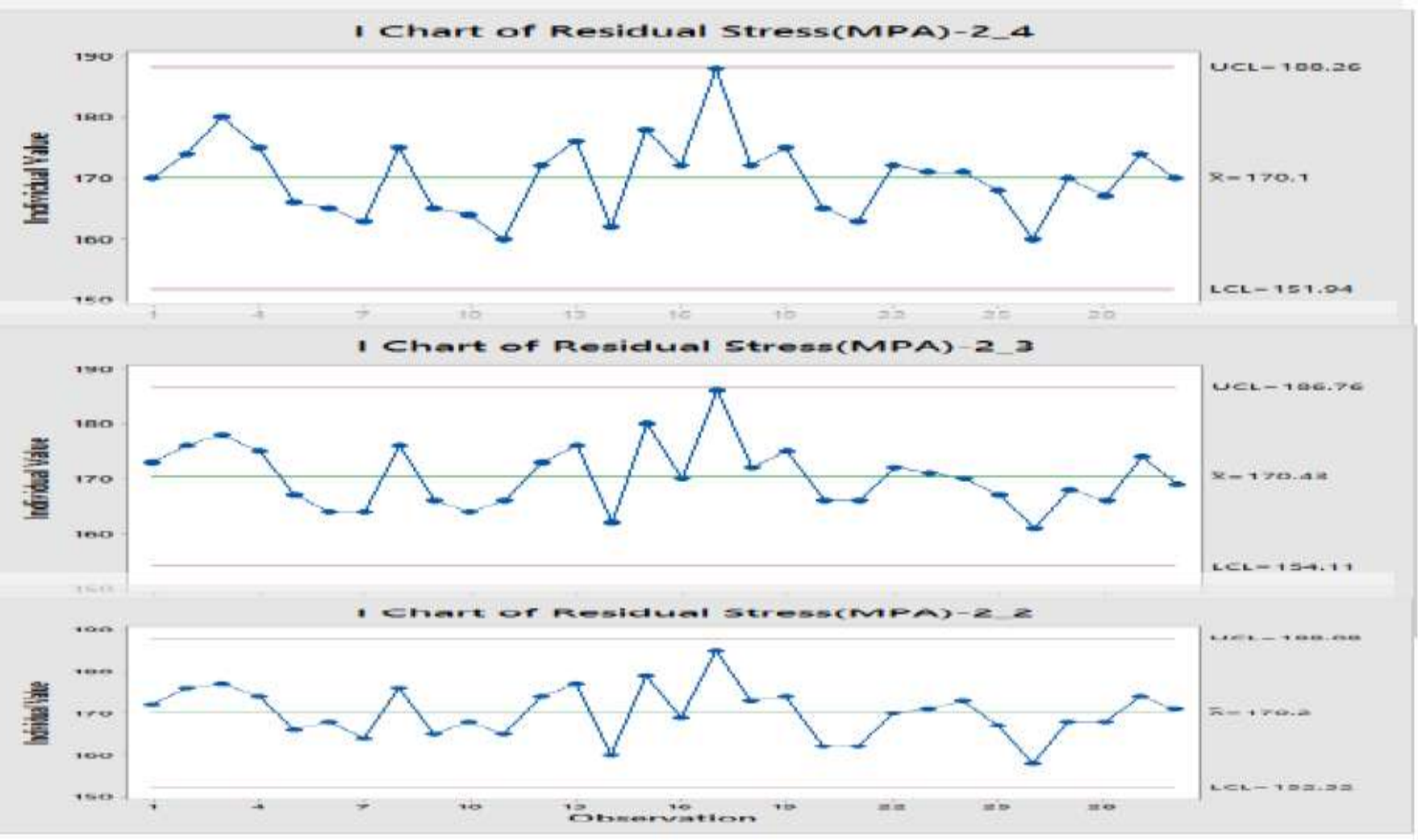

\subsection{Improve Phase}

\section{Future State Value Stream Mapping}

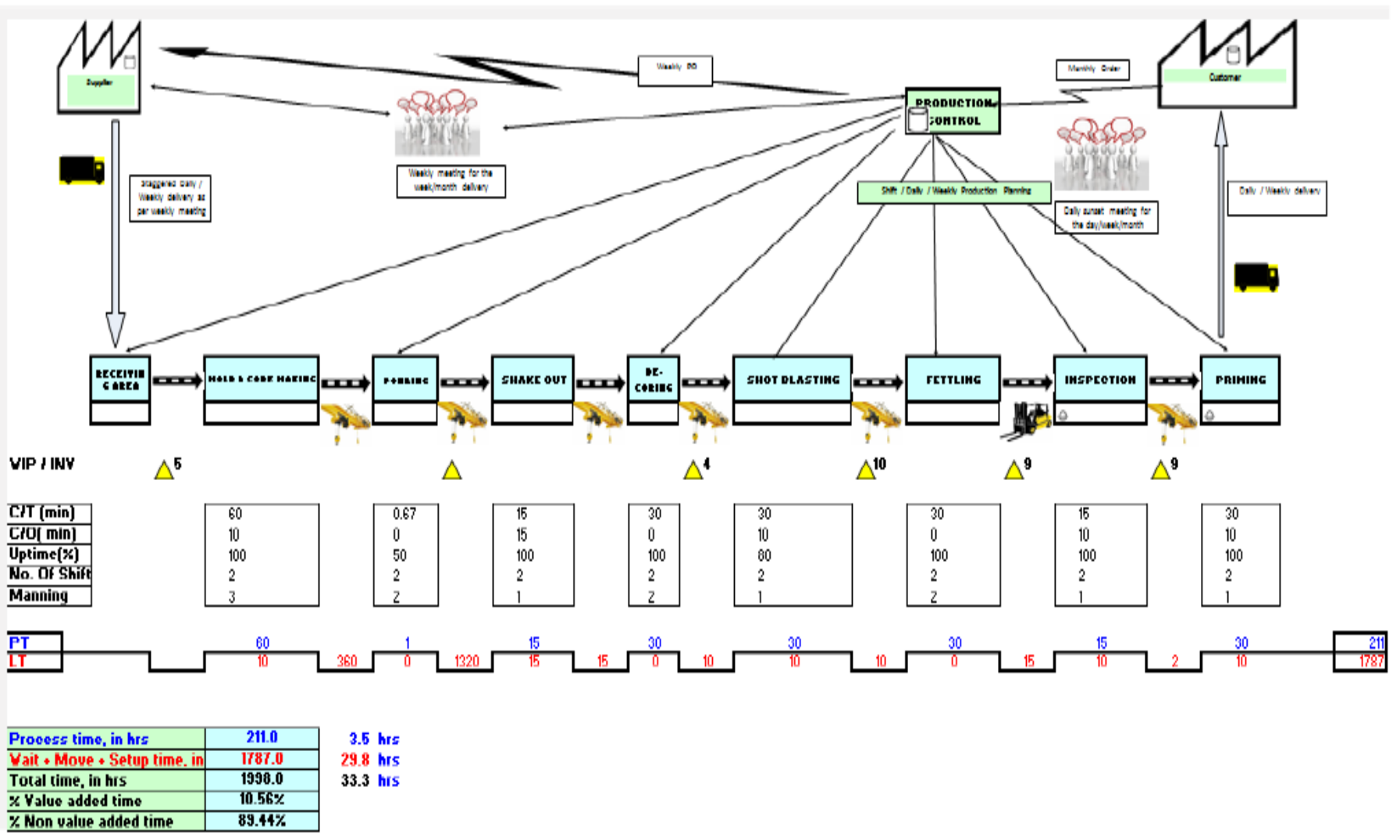




\begin{tabular}{|l|c|}
\hline Process time, in hrs & $\mathbf{2 1 1 . 0}$ \\
\hline Wait + Move + Setup time, in hrs & 1787.0 \\
\hline Total time, in hrs & $\mathbf{1 9 9 8 . 0}$ \\
\hline \% Value added time & $\mathbf{1 0 . 5 6 \%}$ \\
\hline \% Non value added time & $\mathbf{8 9 . 4 4 \%}$ \\
\hline
\end{tabular}

3.5 hrs

29.8 hrs

33.3 hrs

\section{Process Fmea}

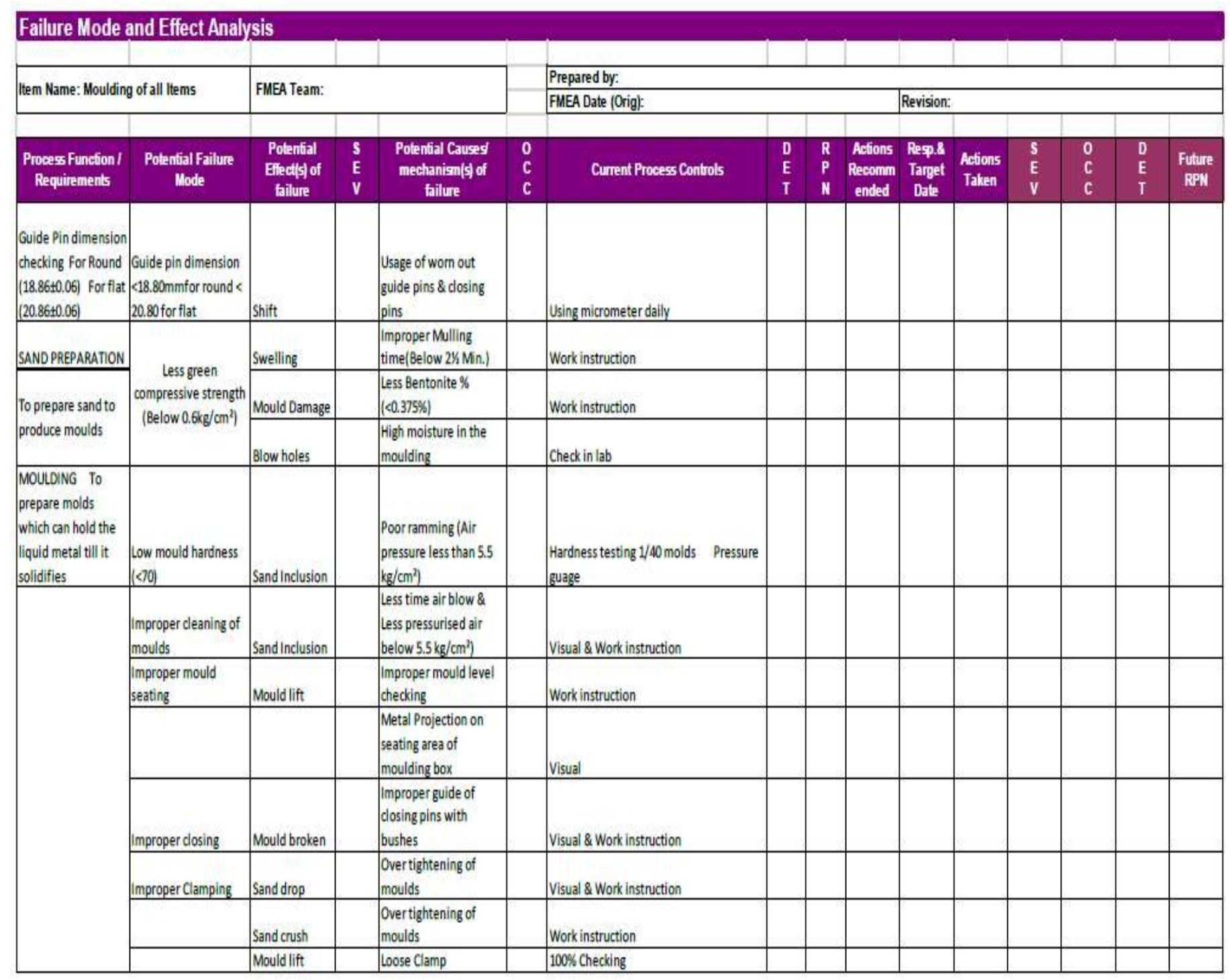




\subsection{Control Phase}

\section{Control Plan}

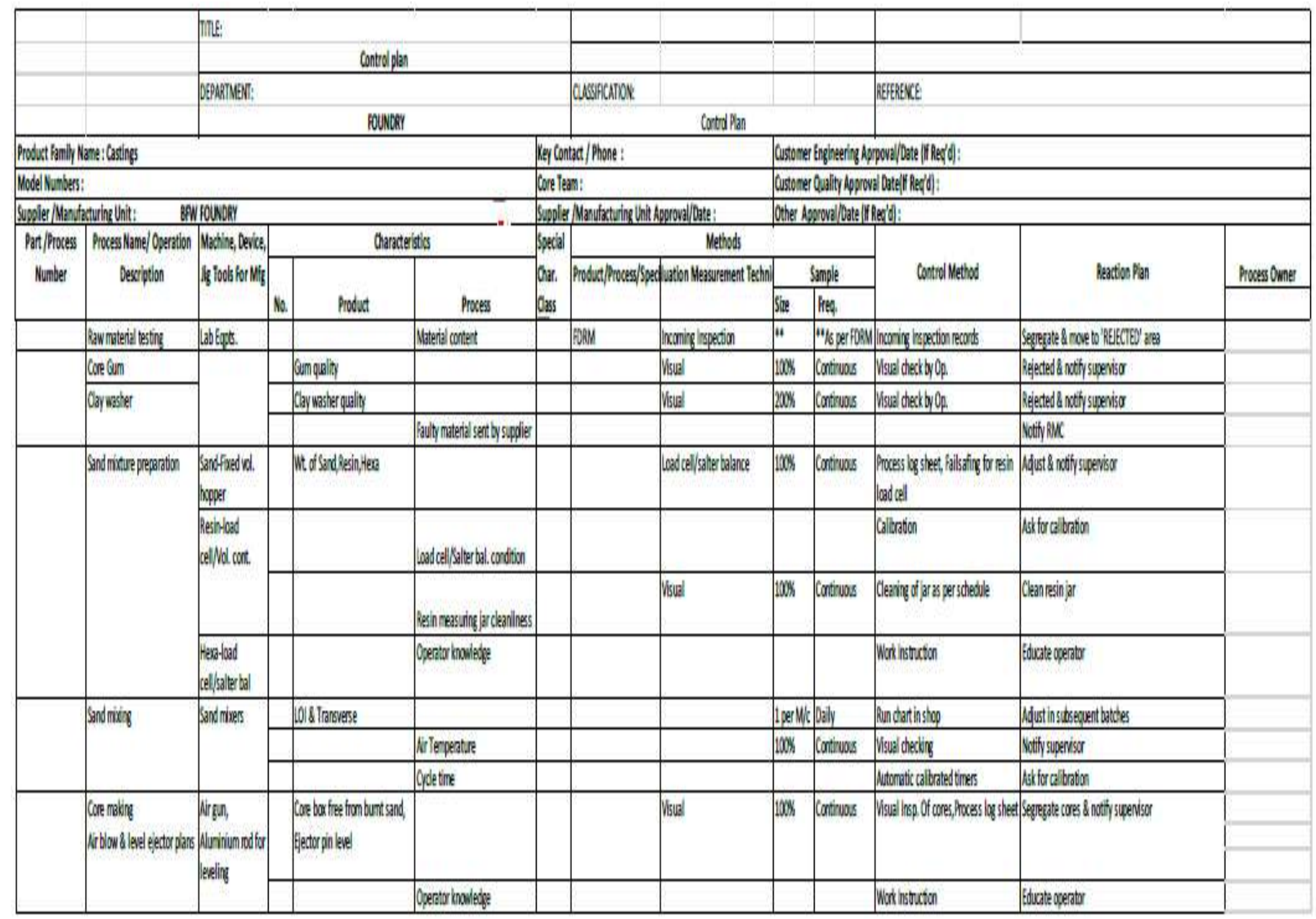

\section{CONCLUSION}

- $\quad$ Projected Cost Savings

\section{FOUNDRY PROJECT COST SAVINGS}

\begin{tabular}{|l|l|r|r|r|r|}
\hline & & VOLUME (2014-15) in metric ton & TOTAL COST & VOLUME(2015-16)in metric ton & TOTAL COST \\
\hline \multirow{2}{*}{ ELIMINATION OF STRESS RELEIVING } & Supplier-2 (1572 Tonnes) at RS 4.50/kg & 1572 & 7073847 & 2360 & 10620000 \\
\cline { 2 - 6 } & Supplier-2 (1522 Tonnes) at Rs 4.50/kg & 1522 & 6085000 & & \\
\hline & & & & \\
\hline
\end{tabular}

- $\quad$ The lead time was reduced from 40.4 hours to 30.3 hours

- The shrinkage defect was eliminated in HSTC table after the grade was changed to $\mathrm{G} 4$.

- Process FMEA and Process Control Plan was developed to control the process.

\section{REFERENCES}

[1]. Dandong Fuding Engineering Machinery Co., Ltd

[2]. Rother, Mike; Shook, John (2003). Learning to see: value-stream mapping to create value and eliminate muda. Brookline, MA: Lean Enterprise Institute. ISBN 09667843-0-8

[3]. FACTA UNIVERSITATIS Series: Economics and Organization Vol. 6, No 1, 2009 
[4]. Lummus, R. R., Robert. J. V. and Rodeghiero, B.,(2006), "Improving Quality through Value Stream Mapping : A Case Study of a

Physician „, s Clinic", Total Quality Management Vol.17, pp.1063- 1075

[5]. Lian, Y. and Landeghem, H. Van.,(2002)," An application of simulation and value stream mapping in lean manufacturing" .Proceedings of Euro.Sim. Symposium.

[6]. Lee, B. (2001),-Introduction to value stream", Lean Manufacturing pp.1-5.

[7].

Mr.Girish.C.Pude1,Prof.G.R.Naik2, Dr.P.G.Naik3(2001), "Application of Value Stream Mapping Tools For Process Improvement a Case Study in Foundry - IOSR Journal of Mechanical and Civil Engineering (IOSR-JMCE) Vol.2278-1684, PP: 07-12. 